\title{
The Effects of Laser Energy, Repetition of Pulse Laser, Wavelength, and Temperature on Silver Nano-Particles
}

\author{
F. M. Aldosari ${ }^{1}$, A. M. Azzeer ${ }^{2} \&$ A. M. Hassib ${ }^{2}$ \\ ${ }^{1}$ Physics Department, Applied and Natural Sciences, Prince Sattam Bin Abdulaziz University, Riyadh 11345, \\ Saudi Arabia \\ ${ }^{2}$ Physics and Astronomy Department, College of Science, King Saud University, Riyadh 11451, Saudi Arabia \\ Correspondence: F. M. Aldosari, Physics Department, Applied and Natural Sciences, Prince Sattam Bin \\ Abdulaziz University, Riyadh 11345, Saudi Arabia. E-mail: fah.aldosari@psau.edu.sa
}

Received: August 17, 2018

Accepted: October 20, 2018

Online Published: November 30, 2018

doi:10.5539/apr.v10n6p38

URL: https://doi.org/10.5539/apr.v10n6p38

\begin{abstract}
To study the effects of temperature, wavelength, laser energy, and repetition of pulsed laser on the silver nanoparticles. The laser induced photoacoustic technique has been used to study photoacoustic properties of silver nanoparticles. The study found a direct correlation between the wavelength and the concentration of the photoacoustic signal. Moreover, an indirect correlation was predicted between the temperature and concentration of the photoacoustic signals. The laser energy was found out to be $355 \mathrm{~nm}$ at a constant repetition rate of $2 \mathrm{~Hz}$ and the repetition rate of $2 \mathrm{~Hz}$ was also selected for the entire measurements. The photoacoustic properties of silver nanoparticles were significantly affected by wavelength, temperature, repetition of pulsed laser, and input energy as predicted by the data collected from the study.
\end{abstract}

Keywords: laser energy, pulse laser, wavelength, temperature, photoacoustic technique, silver nanoparticles

\section{Introduction}

The silver nanoparticles exhibit unique properties when they are prepared with different methods and techniques due to which they are of great importance. The silver nanoparticles have been used in various applications such as photosensitive components, surface-enhanced Raman spectroscopy and catalysts. The surface effect of metal nanoparticles is responsible for exhibiting unique properties since most of the atoms in metal are surface atoms (Solangi et al., 2015). The photoacoustic effect is used to study the physical properties of silver nanoparticles. Alexander Graham Bell, during his search for the discovery of wireless communication, discovered the photoacoustic effect (Rahman et al., 2016). The photoacoustic spectroscopy, which is used to analyze the physical properties of silver nanoparticles, can be classified as photothermal technique. The spectroscopy uses the incident light beam to change the thermal state of the sample. The changes in the thermal state can result in the change in temperature, density, or any other measurable properties of the sample. Thus, the pressure wave produced in the sample can be detected with the help of optical methods, sensitive microphone or piezoelectric devices. The techniques utilized for the detection of these changes are called photoacoustic techniques (Sandler, 2017).

The silver nanoparticles are extensively used in good deal of applications including; biomedical application sensors, nanocomposite fabrications, antibacterial applications, and computing. The nanostructured particles are subjected to various factors, which affect the use of silver nanoparticles in various applications. Shape and size of the nanoparticles are the most important aspects, which greatly influence the properties of nanoparticles. Moreover, the fabrication process also imposes significant influence on the properties of the nanoparticles. Thus, by varying the size and shape of the nanoparticles and by altering the processes for the fabrication of silver nanoparticles, the applications of silver nanoparticles were altered.

The study evaluates the size-dependent properties of the silver nanoparticles and also identifies the optical and electronic properties of the AuNPs. The study also develops strategies to understand and control the collective properties of nanoparticles. Furthermore, the effects of ablation parameters and mechanisms were also studied to control the shape and size of the nanoparticles. 


\subsection{Literature Review}

Kim, Park \& Lim (2015) conducted a study in which the characterization, preparation and utilization of the starch nanoparticles was being discussed. Starch is the most well-known biopolymer and exhibit unique physical property. The starch nanoparticles are being extensively utilized in cosmetics, foods, medicines and several other composites. Furthermore, the starch nanoparticles can be used as drugs and bio-functional ingredients. The most up-to-date information regarding the starch nanoparticles were also included in the overview as well as the physiochemical characterization and preparation processes. Thus, nanoparticles are extremely useful in various applications including its extensive use in medicines and food preservation.

Austin et al (2014) also conducted a study in which the use of gold and silver particles was discussed relating to their optical, photothermal and facile surface chemical properties. The study showed that gold and silver particles are heavily used in the biomedical field due to their extensively small size, which makes them capable of penetrating the cellular membranes. The extremely small size of these particles enables them to interact with the biomolecules and causes changes in their chemical and physical properties. The particles are commonly prepared by using the colloidal synthesis technique. Thus, the study concluded that the use of $\mathrm{Au}$ and $\mathrm{Ag}$ in biomedical field is extremely useful for drug delivery and therapeutic applications. Moreover, Li and Chen (2015) also conducted a study to evaluate the use of Ag nanoparticles for photoacoustic imaging.

\section{Methodology}

The laser induced photoacoustic technique has been used for the study of the photoacoustic properties of silver nanoparticles in dispersions. The study evaluates the use of several devices being used in the study and also discusses the optical effect of photoacoustic signal. The preparation and the optical properties of silver nanoparticles are also discussed in this study.

\subsection{Preparation of Silver Nanoparticles}

\subsubsection{Materials}

The materials used are sodium borohydride and silver nitrate. Triple distilled water was also used for the preparation of aqueous solution with the help of ultrapure purification system.

\subsubsection{Synthesis of Nanoparticles}

For the production of silver nanoparticles, sodium borohydride is used in an excess amount for the reduction of ionic silver and for the stability of the AuNPs being formed in the process. The initial concentration of sodium borohydride was taken to be $\left[\mathrm{NaBH}_{4}\right] /\left[\mathrm{AgNO}_{3}\right]=2$, i.e. the amount of sodium borohydride must be twice of silver nitrate. The variation of sodium borohydride from $2.0 \mathrm{mM}$ resulted in the breakdown of the product; whereas, the silver nitrate was kept to be $1.0 \mathrm{mM}$.

\subsection{Experimental Setup}

The study was conducted to evaluate the photoacoustic properties of the silver nanoparticles in dispersion as well as the repetition of laser pulses, wavelength, size, temperature, and effects of laser energy on the silver nanoparticles were also discussed in the study. The experimental setup being used in the study included: YAG laser with the pump energy of $23 \mathrm{~J}$, Output energy of $532 \mathrm{~nm}$, pulse energy of $532 \mathrm{~nm}$, beam divergence of 1 mrad, polarization of $532 \mathrm{~nm}$, and delay of output relative to pump pulse of 154 micro seconds, the third harmonic generator was also used with the laser; a titanium doped sapphire laser was also used to study the correlation between the wavelength and the photoacoustic signal; UV visible spectrophotometer; a holographic grating; deuterium and halogen lamps as radiative sources; a luminescence spectrometer; xenon flash tube as excitation source; mirror, plane mirror, toroidal mirror and spherical mirrors are also being used in the setup. Excitation and emission monochromators are also used in the experiment. Furthermore, Photodiode Amplifier, two channel digital real-time oscilloscope, intensified charge coupled device, Microtrac S3500 Tri-laser particle size analyzer, transmission electron microscopy, and x-ray diffraction were also used in the experimental setup. Rhodamine $6 \mathrm{G}$ dye was used for testing of the calibration of the experimental setup.

\section{Results}

\subsection{Photoacoustic}

Nine samples were created with varying concentrations and different parameters were investigated. The samples were created with following concentrations:

$$
\begin{gathered}
C 0=2.5 * 10^{-3} M \\
C 1=1.25 * 10^{-3} M
\end{gathered}
$$




$$
\begin{gathered}
C 2=6.25 * 10^{-4} M \\
C 3=3.125 * 10^{-4} M \\
C 4=1.56 * 10^{-4} M \\
C 5=7.81 * 10^{-5} M \\
C 6=3.9 * 10^{-5} M \\
C 7=1.95 * 10^{-5} M \\
C 8=9.76 * 10^{-6} M
\end{gathered}
$$

Several experimental conditions and parameters were studied by conducting photoacoustic investigation of the silver nanoparticles. The results were obtained from the photoacoustic signal from the silver nanoparticles which are demonstrated in figure 1.

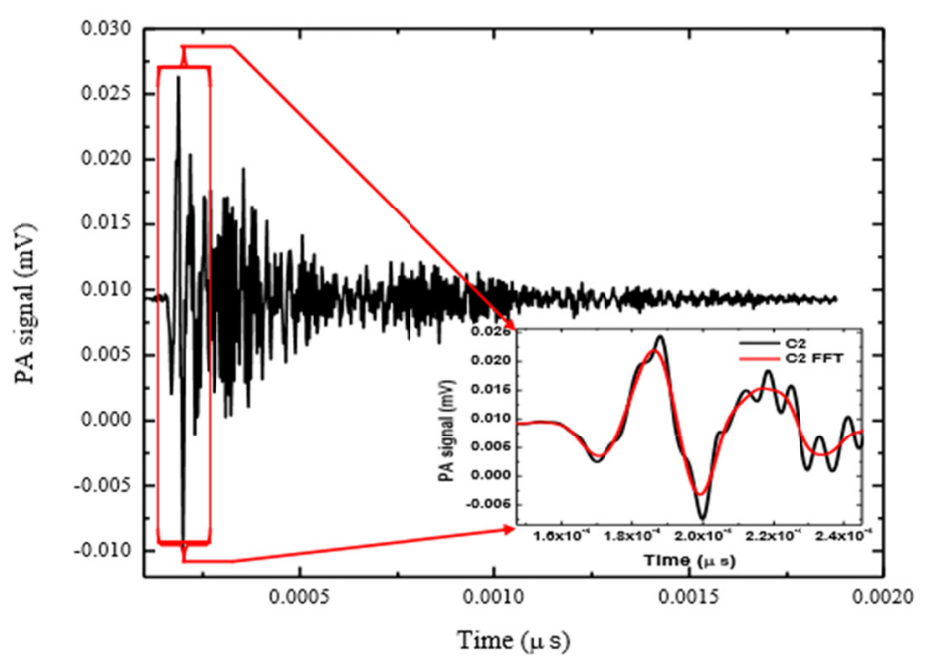

Figure 1. PA signal of silver nanoparticles $(\mathrm{C} 2)$ by oscilloscope at $\mathrm{R}(\mathrm{R}=2 \mathrm{~Hz}, \mathrm{E}=30 \mathrm{~mJ}$

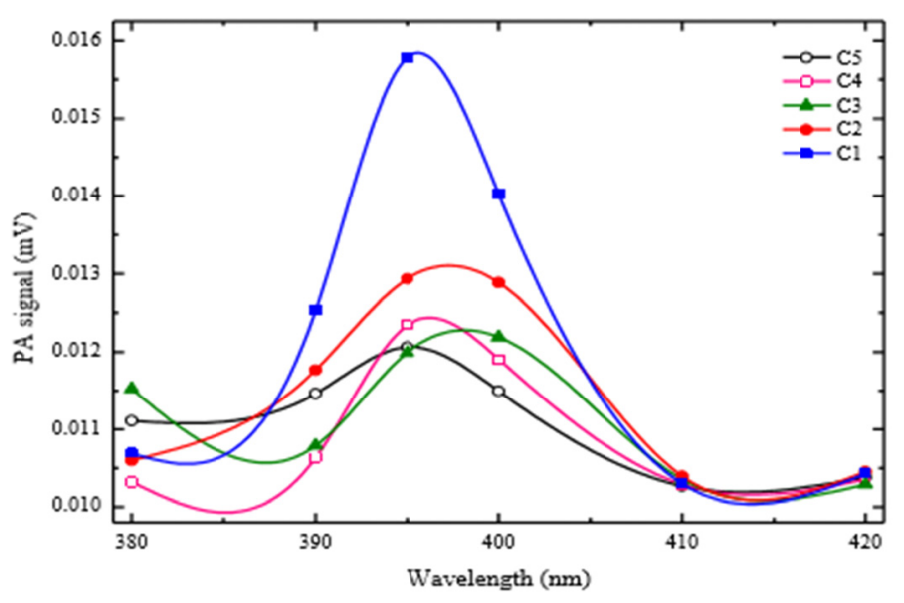

Figure 2. Wavelength at $\mathrm{R}(\mathrm{r})=10 \mathrm{~Hz}, \mathrm{E}=1 \mathrm{~mJ}$ vs. PA signal

\subsection{Study of the Effects of Wavelength}

The silver nanoparticles solution was pumped with the help of Ti-sapphire laser. At constant incident energy of 1 $\mathrm{mJ}$ and constant repetition rate of $10 \mathrm{~Hz}$, the wavelengths $(\lambda)$ were changed from $380 \mathrm{~nm}$ to $420 \mathrm{~nm}$ for the samples of concentrations $\mathrm{C} 1$ to $\mathrm{C} 5$. The results suggested that as the concentration of the solution of the silver nanoparticles was increased, the corresponding PA signal also increased i.e. there was a direct relation found 
between the two parameters. Except for C3, the maximum PA signal peak occurred at nearly $395 \mathrm{~nm}$. Hence, the laser source used with a wavelength of $355 \mathrm{~nm}$, which is a laser source of third harmonic generation, is the best choice.

\subsection{Study of the Effects of Laser Energy}

The samples of silver nanoparticles solution with varying concentrations were investigated to study the effect of the laser energy on the PA signal. To conduct these analyses, the laser input energy was varied from $5 \mathrm{~mJ}$ to 30 $\mathrm{mJ}$ for the concentrations from $\mathrm{C} 1$ to $\mathrm{C} 5$. The laser input energy was of the wavelength of $355 \mathrm{~nm}$ at a constant repetition rate $2 \mathrm{~Hz}$. The results obtained are shown in Figure 3.

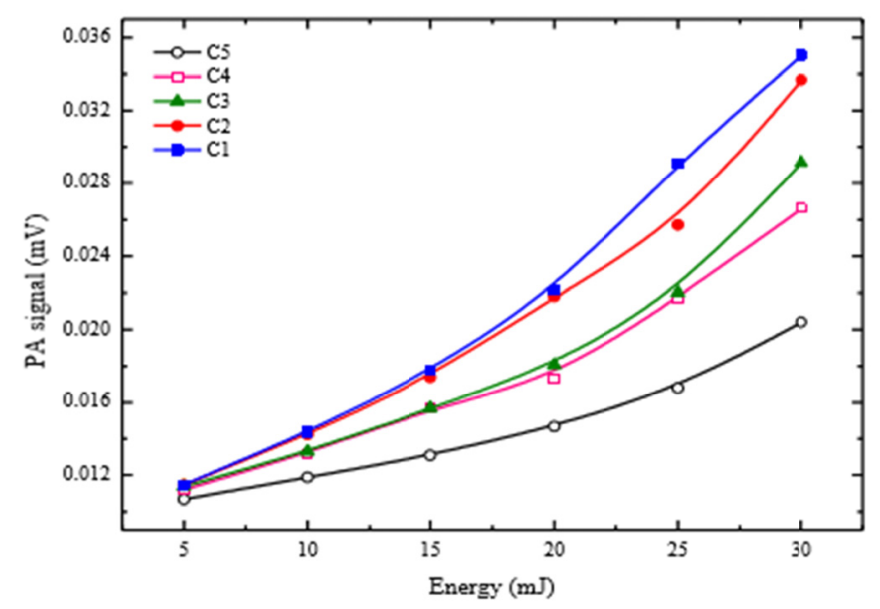

Figure 3. Energy at R(r)=2 Hz vs.PA signal

\subsection{Study of the Effects of Repetition of Pulse Laser}

The relationship and the dependency of the PA signal from different concentrations of the solution of silver nanoparticles and the repetition rate of the laser pulses was studied. The results obtained are shown in Figure 4. The repetition rate of $2 \mathrm{~Hz}$ was chosen for the entire measurements because of the occurrence of best PA signals at this value.

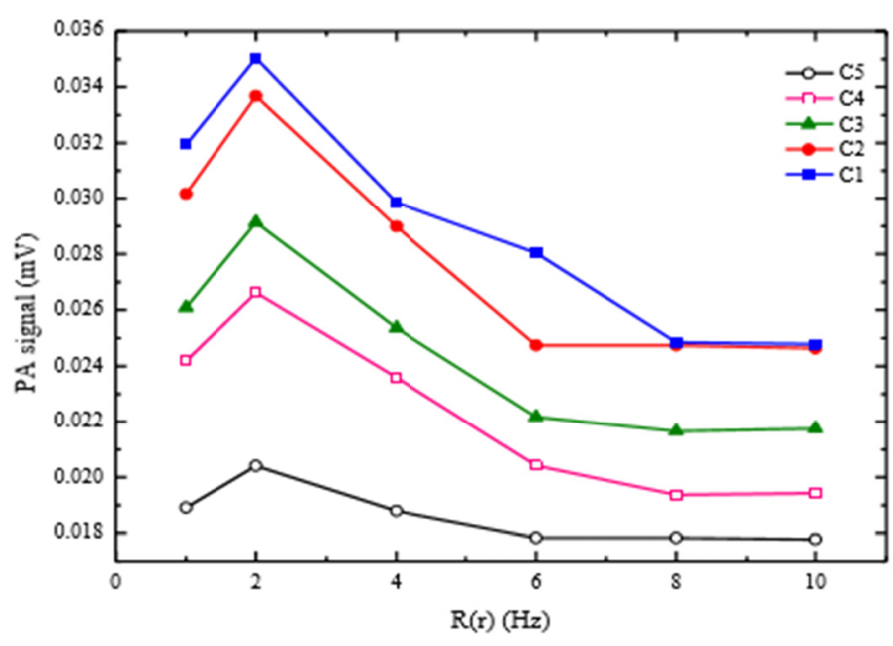

Figure 4. R(r) at $E=30 \mathrm{~mJ}$ vs. PA signal

\subsection{Study of the Effects of Temperature}

The effect of the changing temperature on different concentrations of samples of the silver nanoparticles on the PA signals were studied. The temperature of the silver nanoparticles was varied from $30^{\circ} \mathrm{C}$ to $80^{\circ} \mathrm{C}$ for $\mathrm{C} 1$ to $\mathrm{C} 5$ 
sample concentrations. The following samples were at a repetition rate of $2 \mathrm{~Hz}$ and energy $20 \mathrm{~mJ}$ with laser excitations of wavelength $355 \mathrm{~nm}$. The results suggested an indirect relationship between the PA signal and the temperature i.e. as the temperature was increased, the PA signal was decreased and vice versa. As the temperature of the solution increased, the particles in the solution of silver nanoparticles started to move more rapidly. Moreover, the PA signal was found out to be decreasing for the first, second, third and fourth concentrations, which suggests that the particles of these concentrations were not aggregated. However, particles in the fifth concentration might be aggregated and the separation of the particles from each other is attempted by using the part of the heat. Hence, cooled water was used for the attainment of strong PA signal.

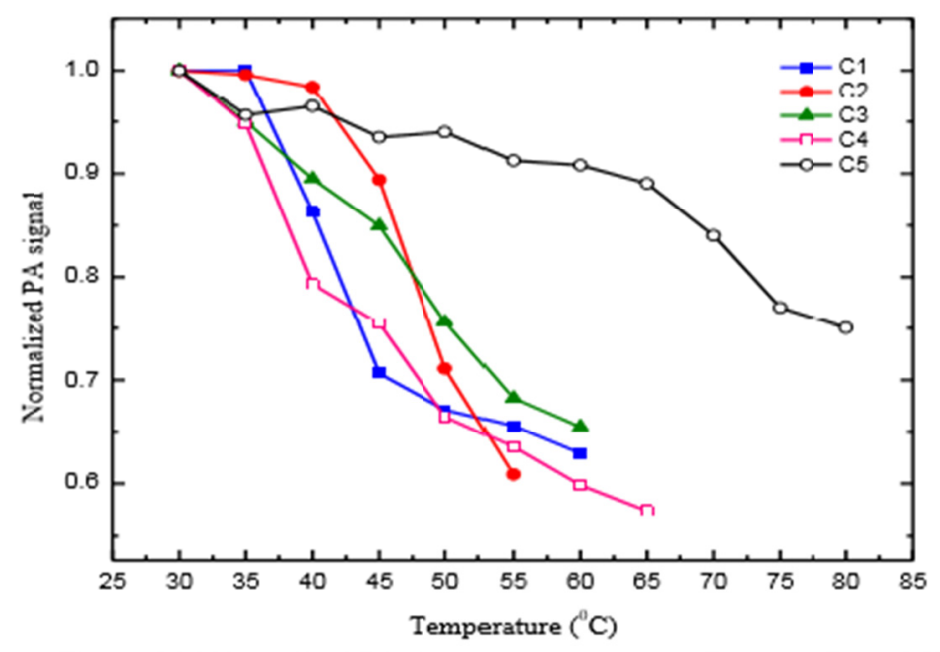

Figure 5. Temperature at $\mathrm{E}=20 \mathrm{~mJ}, \mathrm{R}(\mathrm{r})=2 \mathrm{~Hz}$ vs. Normalized PA signal

\section{Discussion}

The findings have shown that the corresponding PA signal increased as the concentration of the solution of the silver nanoparticles increased. Similarly, the effect of the laser energy on the PA signal varies with respect to the silver nanoparticles solution. In addition, the study has shown that PA signals were occurred based on the repetition rate of $2 \mathrm{~Hz}$ for the entire measurements. These findings have been supported by previous studies. For instance, Cao et al. (2015) conducted a study to evaluate the impact of wavelength and temperature on the photoacoustic signals. A brief model was proposed to study the effects of temperature and wavelength on the photoacoustic signal. The results obtained from the study suggested that the photoacoustic signals varied as the temperature was raised from $8^{\circ} \mathrm{C}$ to $20^{\circ} \mathrm{C}$ and the wavelength was changed from $750 \mathrm{~nm}$ to $850 \mathrm{~nm}$. The present study has analyzed the effects of temperature using $355 \mathrm{~nm}$ and $80^{\circ} \mathrm{C}$ temperature.

In a study conducted by Ren et al. (2017), the effects of pulsed laser parameters on the photoacoustic detection of glucose aqueous solution were investigated. In this study, the photoacoustic detection system was established based on a Q switched Nd: YAG $532 \mathrm{~nm}$ pulsed laser and an ultrasonic detector. At wavelengths from 1300nm to $2200 \mathrm{~nm}$ and different output energy of pulsed laser, the photoacoustic peak values of pure water and glucose were investigated. The results of the study showed that the photoacoustic peak-to-peak values of glucose increased linearly as the output energy of the pulsed laser was increased. Moreover, an exponential decrease was noticed in the output energy of pulsed laser as the wavelengths of the pulsed laser increased, which accounts for the photoacoustic peak-to-peak values.

Another study conducted by Jeon et al. (2018) investigated the effect of laser pulse width on the production of nanoparticles by the fragmentation of laser. The findings of the study showed that the size of the average nanoparticles was found to decrease with decrease in the width of the laser pulse. The rate of increase of the particle size in the nanosecond region was found to be more than the particle size in the picosecond and femtosecond laser pulse widths. Moreover, from the results, it can be deduced that the occurrence of fragmentation was more prominent when shorter pulse width was present.

The study conducted by Wackerow and Abdolvand (2014) studied the generalization of silver nanoparticles with controlled size and partial distribution by pulsed laser irradiation of silver ion-doped glass. In the study, the irradiation of the silver ion exchanged glasses was carried out by picosecond and nanosecond laser pulses. The 
study conducted an optical transmission analysis in which the surface plasmon absorption bands of the silver nanoparticles were demonstrated and showed that it increased with increase in the pulse energies. The thermal analysis conducted in the study showed similar results at the microscopic level for both ps and ns pulses to a temperature distribution which was similar for both. The absorption length was found out to be decreasing with the increase in temperature and the results for ns and ps suggested a very short absorption length at the surface absorption.

The combination of laser therapy with metal nanoparticles in cancer treatment originates from epithelial tissues (Fekrazad et al., 2016). Cancer treatment with the help of laser and nanoparticles has gained enormous attention in the past few decades. The combined use of nanoparticle and laser was investigated in the study for the death of cancerous cells. The type of laser which was used in different investigations NIR which had a wavelength spectrum of $785 \mathrm{~nm}$ up to $1046 \mathrm{~nm}$; whereas, $808 \mathrm{~nm}$ wavelength was used more rapidly and frequently than others. Another wavelength which was investigated and was found in the visible spectra was $420 \mathrm{~nm}$ and up to $690 \mathrm{~nm}$. However, there are few disadvantages of using combined therapy due to difficulty in finding identical particles and high cost of this therapy. Moreover, combined therapy requires high and advanced technology which is difficult to achieve.

Another study conducted by Simandoux et al. (2015) investigated the thermal-based nonlinear photoacoustic generation to differentiate between the different types of absorbing particles. The study focused on the photoacoustic generation from the solutions of gold nanospheres and dye molecules with the help of high frequency ultrasound transducer. At an equilibrium temperature, the photoacoustic emission of the gold nanospheres was observed at a certain low influence for an equilibrium temperature of around $4{ }^{\circ} \mathrm{C}$. At this temperature, the photoacoustic effect appeared to vanish in water; thus, suggesting a non-linear emission from the nanosphere solution. Moreover, the photoacoustic amplitude was also studied, which was chosen as a function of equilibrium temperature from $2^{\circ} \mathrm{C}$ to $20^{\circ} \mathrm{C}$. At the whole temperature range, the photoacoustic amplitude was found to be consistent from the gold nanospheres; however, the photoacoustic amplitude was found to vanish at $4^{\circ} \mathrm{C}$. The results of the study suggested that with respect to the high frequency photoacoustic imaging, the difference can be calculated between the nanoparticles and molecular absorber based on nanoscale temperature rises.

The following study aimed at analyzing the effects of laser energy, repetition of pulse laser, wavelength, and temperature on silver nanoparticles. The study utilized nine samples of varying concentrations and different parameters to evaluate their effects. The results of wavelength on the silver nanoparticles suggested a direct relation between the two parameters. The results of the effects of laser energy on the silver nanoparticles showed that the laser input energy was of the wavelength of $355 \mathrm{~nm}$ at a constant repetition rate $2 \mathrm{~Hz}$. Moreover, effects of repetition of pulse laser was also investigated in which the repetition rate of $2 \mathrm{~Hz}$ was selected for the entire measurements due to the occurrence of best PA signals at this specific value. In the end, the effect of temperature was also studied on the silver nanoparticles. The results suggested that the temperature affected the particles in the solution of silver nanoparticles. Moreover, an indirect relationship was predicted between the PA signal and the temperature. The following study have several implications; such as, the study will prove to be beneficial to reduce the errors associated with the photoacoustic signal analysis due to the wavelength and temperature. The study will help the physicists to improve the thermal photoacoustic measurement and imaging quality.

\section{Acknowledgements}

The author is very thankful to all the associated personnel in any reference that contributed in/for the purpose of this research. Further, this research holds no conflict of interest and is not funded through any source.

\section{References}

Austin, L. A., Mackey, M. A., Dreaden, E. C., \& El-Sayed, M. A. (2014). The optical, photothermal, and facile surface chemical properties of gold and silver nanoparticles in biodiagnostics, therapy, and drug delivery. Archives of toxicology, 88(7), 1391-1417.

Cao, Y., Xiang, Y., Jiao, Y., Han, Z., Jian, X., \& Cui, Y. (2015, June). Effect of temperature and wavelength on photoacoustic signal. In Photonics for Energy (pp. JW3A-45). Optical Society of America.

Fekrazad, R., Naghdi, N., Nokhbatolfoghahaei, H., \& Bagheri, H. (2016). The combination of laser therapy and metal nanoparticles in cancer treatment originated from epithelial tissues: a literature review. Journal of lasers in medical sciences, 7(2), 62. 
He, Y., Du, Z., Ma, S., Liu, Y., Li, D., Huang, H., ... \& Zheng, X. (2016). Effects of green-synthesized silver nanoparticles on lung cancer cells in vitro and grown as xenograft tumors in vivo. International journal of nanomedicine, 11, 1879.

Jeon, J. W., Yoon, S., Choi, H. W., Kim, J., Farson, D., \& Cho, S. H. (2018). The Effect of Laser Pulse Widths on Laser-Ag Nanoparticle Interaction: Femto-to Nanosecond Lasers. Applied Sciences, 8(1), 112.

Kim, H. Y., Park, S. S., \& Lim, S. T. (2015). Preparation, characterization and utilization of starch nanoparticles. Colloids and Surfaces B: Biointerfaces, 126, 607-620.

Li, W., \& Chen, X. (2015). Gold nanoparticles for photoacoustic imaging. Nanomedicine, 10(2), 299-320.

Rahman, T., Adams, A. T., Schein, P., Jain, A., Erickson, D., \& Choudhury, T. (2016, November). Nutrilyzer: A Mobile System for Characterizing Liquid Food with Photoacoustic Effect. In Proceedings of the 14th ACM Conference on Embedded Network Sensor Systems CD-ROM (pp. 123-136). ACM.

Ren, Z., Liu, G., Huang, Z., \& Zeng, L. (2017, October). The effects of pulsed laser parameters on the photoacoustic detection of glucose aqueous solution. In AOPC 2017: Laser Components, Systems, and Applications (Vol. 10457, p. 1045702). International Society for Optics and Photonics.

Sandler, S. I. (2017). Chemical, biochemical, and engineering thermodynamics. John Wiley \& Sons.

Simandoux, O., Prost, A., Gateau, J., \& Bossy, E. (2015). Influence of nanoscale temperature rises on photoacoustic generation: Discrimination between optical absorbers based on thermal nonlinearity at high frequency. Photoacoustics, 3(1), 20-25.

Solangi, K. H., Kazi, S. N., Luhur, M. R., Badarudin, A., Amiri, A., Sadri, R., ... \& Teng, K. H. (2015). A comprehensive review of thermo-physical properties and convective heat transfer to nanofluids. Energy, 89 , $1065-1086$.

Wackerow, S., \& Abdolvand, A. (2014). Generation of silver nanoparticles with controlled size and spatial distribution by pulsed laser irradiation of silver ion-doped glass. Optics express, 22(5), 5076-5085.

\section{Copyrights}

Copyright for this article is retained by the author(s), with first publication rights granted to the journal.

This is an open-access article distributed under the terms and conditions of the Creative Commons Attribution license (http://creativecommons.org/licenses/by/4.0/). 